\title{
Distortions of depth-order relations and parallelism in structure from motion
}

\author{
FULVIO DOMINI and CORRADO CAUDEK \\ Cognitive Technology Laboratory, Trieste, Italy \\ and \\ SCOTT RICHMAN \\ University of California, Irvine, California
}

\begin{abstract}
Four experiments related human perception of depth-order relations in structure-from-motion displays to current Euclidean and affine theories of depth recovery from motion. Discrimination between parallel and nonparallel lines and relative-depth judgments was observed for orthographic projections of rigidly oscillating random-dot surfaces. We found that (1) depth-order relations were perceived veridically for surfaces with the same slant magnitudes, but were systematically biased for surfaces with different slant magnitudes. (2) Parallel (virtual) lines defined by probe dots on surfaces with different slant magnitudes were judged to be nonparallel. (3) Relative-depth judgments were internally inconsistent for probe dots on surfaces with different slant magnitudes. It is argued that both veridical performance and systematic misperceptions may be accounted for by a heuristic analysis of the first-order optic flow.
\end{abstract}

Appropriate 2-D motions produce phenomenal impressions of movement in depth (see, e.g., Miles, 1931; Musatti, 1924; Wallach \& O'Connell, 1953). Certain types of these phenomena have been named structure from motion (SFM). The questions of how these impressions arise and what type of geometric structure is derived from these motions have led to both experimental and theoretical work on depth recovery from motion. The psychophysical research has evaluated the capabilities of the human visual system in light of the constraints and the scope of the algorithms devised to derive 3-D geometric properties from 2-D motions (for a review, see Braunstein, 1989; Norman \& Todd, 1992).

\section{Euclidean and Affine Theories of SFM}

The analysis of the 2-D motions compatible with the orthographic projection of rigid 3-D motion allows the recovery of metric, affine, or purely local properties of the projected objects. Ullman's theorem, for example, demonstrates that three orthographic views of four points undergoing rigid motion are sufficient to determine the relative depths of the points uniquely (under the assumption of rigid motion), and hence the full metric Euclidean structure thereof (Ullman, 1979). Consistent with this theorem, Ullman proposed that the perceptual recovery of depth should also be unique and veridical for multipleview display and that subjects' abilities to perform metric judgments in SFM displays should improve with increasing numbers of views (Ullman, 1984). We term this

Correspondence should be addressed to F. Domini, Cognitive Technology Laboratory, c/o INSIEL SpA, AREA Science Park, Padriciano 99,34012 Trieste, Italy (e-mail: domini@aris.ss.uci.edu). approach the Euclidean theory of depth recovery from motion. Some evidence supporting this theory has been provided by Hildreth, Grzywacz, Adelson, and Inida (1990; but see also Norman \& Todd, 1993).

A competing theory is that projected 3-D shape is not uniquely recovered by the perceptual system from both two- and multiple-view displays, but rather is established only up to a family of affine transformations (Koenderink \& van Doorn, 1991; Norman \& Todd, 1993; Todd \& Bressan, 1990). This family entails linear transformations that change the $z$-coordinates but leave the $x$-and $y$ coordinates of the points fixed. We term this approach the affine theory of depth recovery from motion. Support for this theory has been provided by the finding that performance in psychophysical tasks requiring the knowledge of Euclidean structural relations is poorer than in tasks requiring only knowledge of affine properties, and by the finding that perceptual performance does not improve if additional views are added to a two-view display (Liter, Braunstein, \& Hoffman, 1993; Todd, Akerstrom, Reichel, \& Hayes, 1988; Todd \& Bressan, 1990; Todd \& Norman, 1991; but see also Eagle \& Blake, 1995; Pollick, 1997).

Three common properties are shared by both Euclidean and affine theories of depth recovery from motion: (1) The ordinal structure of the projected shape is preserved in the recovered 3-D shape. Although this is an obvious requirement for any Euclidean algorithm, Koenderink and van Doorn (1991) showed that it also remains true for an affine algorithm of depth recovery from motion. (2) Lines and planes that are parallel for the projected shape also remain parallel for the recovered 3-D shape. Any affine transformation, in fact, preserves parallelism (see Koenderink \& van Doorn, 1991). (3) The ordinal structure of the recovered 3-D shape is internally consistent. This 


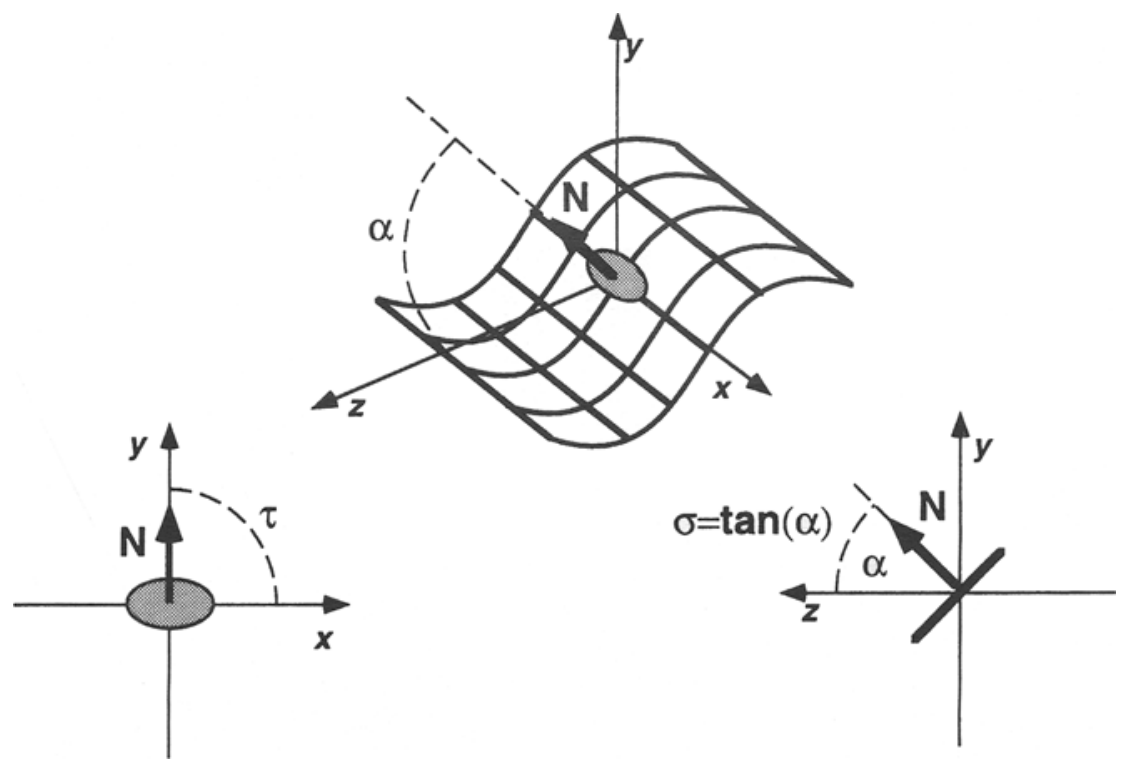

Figure 1. Top: A generic 3-D smooth surface can be locally approximated by a planar patch with normal $\mathrm{N}$. Bottom left: $x, y$ view of the local patch. Tilt $(\tau)$ is defined as the angle between the $x, y$ projection of the normal to the patch and the $x$-axis. Bottom right: $y, z$ view of the local patch. Slant $(\sigma)$ is defined as the tangent of the angle $(\alpha)$ between the normal to the patch and the $z$-axis.

follows from the fact that both Euclidean and affine algorithms preserve the ordinal structure of the projected object. If the symbol " $<$ " is taken to mean "closer in depth than," and the symbol " $>$ " is taken to mean "further in depth than," then for points $\mathrm{P}_{0}, \mathrm{P}_{1}, \ldots, \mathrm{P}_{\mathrm{i}}, \ldots, \mathrm{P}_{\mathrm{n}}$ two conditions must be satisfied according to the usual definition of ordinal relation: (1) $P_{i}>P_{j} \Rightarrow \neg\left(P_{j}>P_{i}\right)$, and $(2)\left(P_{i}>P_{j}\right) \wedge\left(P_{j}>P_{k}\right) \Rightarrow P_{i}>P_{k}$. From this definition it follows that the judgments of the perceived order of depth cannot be of the sort: $\left(\mathrm{P}_{0}>\mathrm{P}_{1}\right) \ldots \wedge\left(\mathrm{P}_{\mathrm{i}}>\mathrm{P}_{\mathrm{i}+1}\right)$ $\ldots \wedge\left(P_{n}>P_{0}\right)$. In fact, this would imply that $\left(P_{0}>P_{i}\right) \wedge$ $\left(P_{i}>P_{0}\right)$. In turn, this violates Condition 1 and makes the ordinal structure of the recovered 3 -D shape internally inconsistent.

The purpose of the present paper was to explicitly test all of these necessary conditions for both Euclidean and affine theories of the perceptual recovery of depth from motion. First, however, we discuss the properties of a local invariant of the first-order optic flow and the ensuing theory that motivates the present experiments.

\section{Local Linear Optic Flow}

A generic smooth 3-D surface can be locally approximated by a planar patch. The orientation of a planar surface in 3-D space is usually described in terms of two parameters: slant $(\sigma)$ and tilt $(\tau)$. Slant is defined as the tangent of the angle between the normal to the patch and the $z$-axis; tilt is defined as the angle between the projection of the normal and the $x$-axis (Figure 1). The optic flow produced by the (perspective or orthographic) projection of a planar surface undergoing a generic 3-D motion gives rise to a local linear velocity field. The local instantaneous flow of a continuous velocity field is customarily divided into four components: def, curl, transl, and div. The differential invariants of the optic flow correspond to a pure local shape change without a change in area (def or shear), a local rotation (curl), a translation (transl), and an isotropic expansion or contraction (div) (see Koenderink, 1986).

Both perspective and orthographic analyses interpret a linear velocity field as the projection of a planar surface moving in 3-D space. The orientation of this planar surface, however, cannot be uniquely recovered from the first-order velocity field. For a perspective analysis, this ambiguity arises from the fact that the projection of planar patches having different slants and rotating and translating by different amounts gives rise to identical velocity fields. A particular case of this situation occurs when the projected motion is produced solely by a translation (motion parallax). In this particular case, however, the orientation of the projected patch and its translation velocity can be uniquely and veridically recovered from the linear velocity field if the interpretation process assumes that the angular velocity component is equal to zero (see, e.g., Braunstein, Hoffman, Shapiro, Andersen, \& Bennett, 1987). For an orthographic analysis, on the other hand, the linear velocity field is always ambiguous. Hoffman (1982), in fact, has shown that in terms of an orthographic analysis, the first-order optic flow is sufficient for the veridical derivation (up to a reflection) of the tilt of the axis of rotation $\left(\tau_{\text {axis }}\right)$, the component about the $z$-axis $(\rho)$ of the projected angular velocity, and the tilt of a planar patch $(\tau)$. The first-order optic flow, however, is insufficient to determine veridically the slants $(\sigma)$ of a planar 
patch and the component parallel to the $x, y$ plane $(\omega)$ of the projected angular velocity. Infinite combinations of $\sigma$ and $\omega$, in fact, give rise to the same magnitude of $d e f$ :

$$
d e f=\sigma \omega .
$$

The first-order optic flow, moreover, is insufficient to veridically derive the slant of the axis of rotation $\left(\sigma_{\mathrm{axis}}\right)$. This parameter of the projected motion is also, in fact, a function of $\omega$ :

$$
\sigma_{\text {axis }}=\arctan \left(\frac{\omega}{\rho}\right)
$$

The veridical recovery of the slant of the axis of rotation, the slant of a planar patch, and the component parallel to the $x, y$ plane of the projected angular velocity require the use of the second temporal derivatives of the velocity field and the introduction of further assumptions (e.g., rigidity, rotation at a constant angular velocity, smoothness of the velocity field) in the interpretation process (see Bennett, Hoffman, Nicola, \& Prakash, 1989; Koenderink, 1986; Koenderink \& van Doorn, 1975, 1976; Longuet-Higgins \& Prazdny, 1980; Prazdny, 1980; Ullman, 1979, 1983).

In summary, a linear velocity field does not allow a oneto-one mapping between the projected stimulus properties and the distal parameters (i.e., the local slant, $\sigma$, and the angular velocity, $\omega$ ). In general, this ambiguity is true for both perspective and orthographic analyses of the first-order optic flow. ${ }^{1}$

\section{Heuristic Derivation of Slant \\ From First-Order Optic Flow}

We propose that even though the first-order velocity field is not sufficient for uniquely and veridically recovering the projected slant of a planar surface, perceived slant is nevertheless recovered from the first-order properties of a velocity field. Evidence supporting this hypothesis has been provided by Domini, Caudek, and Gerbino (1995) and by Domini and Caudek (in press). Domini and Caudek presented observers with the orthogonal projection of oscillating random-dot planar surfaces. A transparent hemisphere was simulated within a circular gap of the multiview stimulus displays, and the observers were asked to manipulate the slant of the base of the hemisphere and its amplitude of rotation so that the base of the hemisphere appeared to be parallel to the perceived surface for the whole duration of each oscillation cycle. Domini and Caudek found that perceived slant magnitudes were an increasing sublinear function of $d e f$ and were not significantly influenced by the simulated slant magnitudes.

We reasoned that if perceived slant of a surface $\left(\sigma^{\prime}\right)$ is a nonlinear function of def, the integration of the local slant magnitudes should produce systematic distortions in the reconstruction of the global 3-D shape that cannot be accounted for by either Euclidean or affine theories of depth recovery from motion. These hypothesized distor- tions in the recovered 3-D shape can be described by considering the depth separation of two points on a planar surface. If $\sigma^{\prime}$ is a sublinear function of def, the recovered depth separation of two points of the surface must be related to the simulated depth separation according to the following relation (see the Appendix):

$$
z^{\prime}=\omega F(\sigma \omega) z,
$$

where $z^{\prime}$ is the recovered depth separation, $z$ is the simulated depth separation, $\sigma$ and $\omega$ are the simulated surface slant and angular velocity magnitudes, and $F(d e f)$ is equal to the ratio between $f_{\text {sublinear }}(d e f)$ and $\operatorname{def}$. If $f(d e f)$ is a sublinear function, then $F($ def $)$ is a decreasing function of def. On the basis of Equation 3, therefore, we can formulate three hypotheses that will be directly tested in the present paper $^{2}$ (see also Domini \& Braunstein, 1998).

(1) Perceived 3-D shape does not preserve the ordinal structure of the projected shapes. Let us consider two rigidly rotating surfaces with different slant magnitudes (Figure 2, top panel). Let $\mathrm{P}_{0}$ and $\mathrm{P}_{1}$ be two probe dots simulating the same $z$-depth magnitude $(\Delta z)$ relative to $\mathrm{P}_{\mathrm{m}}$ (the point corresponding to the intersection of the two surfaces). According to Equation 3, the probe placed on the surface with the larger simulated slant should be perceived as having the smaller relative $z$-depth $\left(\Delta^{\prime} z_{1}<\Delta^{\prime} z_{0}\right)$. For two surfaces with slants $\sigma_{1}>\sigma_{0}$, in fact, it must be true that $F\left(\omega \sigma_{1}\right)<F\left(\omega \sigma_{0}\right)$, since $\omega$ is constant and $F$ is a decreasing function. From this it follows that $\Delta z_{1}^{\prime}<\Delta z_{0}^{\prime}$. Preservation of depth-order relations was tested in Experiments 1 and 2 .

(2) Perceived orientation of lines and planes does not preserve parallelism of the projected shapes. Let us consider two lines passing through two pairs of dots $\left(\mathrm{P}_{0}, \mathrm{P}_{1}\right.$ and $\left.P_{2}, P_{3}\right)$ placed on the surface arrangement depicted in Figure 2 (bottom panel). If perceived depth is recovered according to Equation 3, then the perceived $z$-depth magnitudes relative to the point $P_{m}$ will be equal for the points $\mathrm{P}_{0}, \mathrm{P}_{1}$, but different for the points $\mathrm{P}_{2}, \mathrm{P}_{3}$. The points $P_{0}, P_{1}$, in fact, are located on surfaces with equal slant magnitudes, whereas the points $\mathrm{P}_{2}, \mathrm{P}_{3}$ are located on surfaces with different slant magnitudes. As a consequence, simulated parallel lines (i.e., the line passing through the points $\mathrm{P}_{0}, \mathrm{P}_{1}$ and the line passing through the points $P_{2}, P_{3}$ ) should be perceived as nonparallel. Preservation of parallelism was tested in Experiment 3.

(3) Perceived ordinal structure is internally inconsistent. Let us consider the corrugated circular crown surface represented in Figure 3. Each corrugation of the circular crown surface is made up of two slanted surfaces arranged as in Figure 2 (top panel). Two probe dots are placed on the two surfaces of each corrugation at the same relative $z$-depth. As noted, Equation 3 predicts a larger recovered relative-depth magnitude for the probe on the surface with the smaller simulated slant. By combining the recovered depth-order relations for the probe pairs placed on each of the corrugations of the circular crown surface, therefore, 


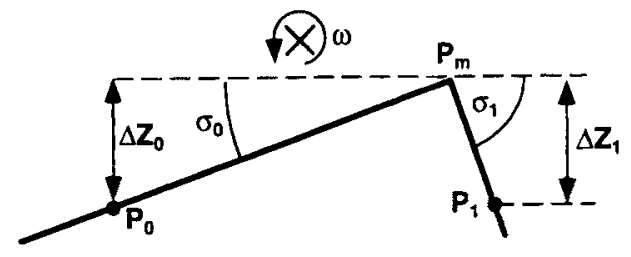

prediction: $\Delta \mathbf{Z}_{0^{\prime}}>\Delta \mathbf{Z}_{1}{ }^{\prime}$

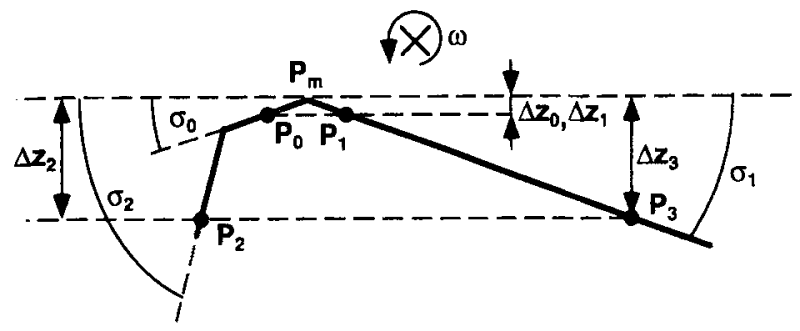

prediction: $\mathrm{P}_{0} \mathrm{P}_{1}{ }_{1} \# \mathrm{P}_{2} \mathrm{P}_{3}$

Figure 2. Top: View from above of two planar surfaces with slants $\sigma_{0}$ and $\sigma_{1}$. The probe dots $P_{0}$ and $P_{1}$ have the same simulated $z$-depth relative to the point $P_{m}\left(\Delta z_{0}=\Delta z_{1}\right)$. Equation 3 predicts that $\Delta z_{0}^{\prime}>\Delta z_{1}^{\prime}$. Bottom: View from above of three planar surfaces with slants $\sigma_{0}, \sigma_{1}$, and $\sigma_{2}\left(\sigma_{0}=\sigma_{1}, \sigma_{2}>\sigma_{1}\right)$. The simulated $z$-depth magnitudes relative to the point $P_{m}$ are the same for $P_{0}$ and $P_{1}\left(\Delta z_{0}=\Delta z_{1}\right)$, and for $P_{2}$ and $P_{3}\left(\Delta z_{2}=\Delta z_{3}\right)$. Equation 3 predicts that $\Delta z_{0}^{\prime}=\Delta z_{1}^{\prime}$, and that $\Delta z_{2}^{\prime}=\Delta z_{3}^{\prime}$.

we should expect a violation of the transitivity principle of the form $P_{0}>P_{1} \ldots>P_{7}>P_{0}$. Internal consistency of depth-order judgments was evaluated in Experiment 4.

In conclusion, the heuristic described by Equation 3 allows us to distinguish between two hypotheses concerning the function relating perceived to simulated slant magnitudes in SFM. According to a first hypothesis, this function is linear. Any (Euclidean or affine) model of depth recovery from motion consistent with this first hypothesis, therefore, will preserve the depth-order relations of the projected image features, will preserve parallelism, and will recover an internally consistent 3-D structure. According to a second hypothesis, perceived slant is a sublinear function of def. For constant simulated angular velocities, therefore, perceived slant will be a sublinear function of simulated slant. As noted, any model of depth recovery from motion consistent with this second hypothesis will not preserve the depth-order relations of the projected image features, will not preserve parallelism, and will recover an internally inconsistent $3-D$ structure.

\section{EXPERIMENT 1}

If perceived slant is a sublinear function of def, it should be possible to construct stimuli for which the perceived ordinal structure does not preserve the ordinal structure of the projected object. This hypothesis was tested by placing probe dots on a dihedral angle made up of two rigidly oscillating planes rendered by random placement of points on each surface and by manipulating def.

\section{Method \\ Subjects. Ten University of Trieste undergraduates participated in this experiment. All of them were naive to the purpose of the ex- periment.}

Apparatus. The displays were presented on a high-resolution color monitor $(1,280 \times 1,024$ addressable locations $)$ under the control of a Silicon Graphics IRIS Workstation. The screen had a refresh rate of $60 \mathrm{~Hz}$ and was approximately photometrically linearized. An anti-aliasing procedure was used: For point-light locations falling on a pixel boundary, the screen luminance was proportionally adjusted in the relevant addressable locations. The graphics buffer was 8 bits deep ( 256 gray levels).

To limit the effectiveness of the flatness cues, subjects viewed the displays through a viewing tube from a distance of $80 \mathrm{~cm}$. The viewing tube limited the visible portion to a circular region of about $10^{\circ}$ of visual angle. The simulated surfaces did not extend beyond the viewing area. The apparatus was the same in all experiments. The displays were viewed with an eyepatch over one eye

Design. Condition (same vs. different slant) and position (probe with the largest distance relative to the axis of rotation to the right or to the left of the stimulus display) were the two independent variables. Each subject viewed 10 presentations in random order of the four different stimuli. Eight additional trials were presented at the beginning of each experimental session in order to familiarize the subjects with the stimulus displays.

Stimuli. The stimuli consisted of high-luminance dots moving on a low-luminance background. The horizontal motions of the dots were computed as simulating an orthographic projection of two surfaces oscillating about a fixed vertical axis contained in the projection plane (Figure 4). Each stimulus display was contained within a rectangular window measuring $7.9^{\circ}$ (width) $\times 1.29^{\circ}$ (height) of visual angle to prevent changes in the projected contours of the simulated surfaces from being visible. The two surfaces were simulated as meeting at a vertical edge, with the position of the axis of rotation bisecting evenly the length of the stimulus window. The simulated surfaces were oriented so as to be parallel to the axis of rotation. The dots were randomly distributed with uniform probability density over the projection plane (not evenly distributed over the simulated surfaces). Average dot density was equal to $4 \mathrm{dots} / \mathrm{cm}^{2}$.

Two conditions were created. In the same-slant condition, at the midpoint of the oscillation sequence the slants of the two simulated surfaces were equal to $.64\left(32.7^{\circ}\right)$; in the different-slant condition, the slants of the two simulated surfaces were equal to 2.74 and .36 $\left(70^{\circ}\right.$ and $\left.20^{\circ}\right)$. The surface with the largest slant was on the right in half the trials and on the left in the remaining half. Two probe dots (high-luminance dots with a diameter of $1.5 \mathrm{~mm}$ ) were positioned on each surface at different depths relative to the rotation axis. The simulated relative depths of the probe dots were the same in both experimental conditions $(2.44$ and $1.58 \mathrm{~cm}$ ). As a consequence, the projected $2-\mathrm{D}$ velocities of the probe dots did not vary across conditions. For the different-slant condition, the probe at a distance of $2.44 \mathrm{~cm}$ from the axis of rotation was always positioned on the surface with the largest slant. The probe positioned at the largest depth was presented on the left surface in half the trials and on the right surface in the remaining half. The difference of $.86 \mathrm{~cm}$ in the simulated $z$-depth magnitudes for the two probe dots was determined by performing a pilot experiment. The goal of the pilot experiment was to find a magnitude of a simulated $z$-depth difference for the two probe dots $(\Delta z)$ that, in the different-slant condition, would give rise to the perception of the following depth-order relation: $z^{\prime}>(z+\Delta z)^{\prime}$.

The projection of the axis of rotation in the $x, y$ plane bisected evenly the distance between the two probe dots in both conditions. In order to keep constant the simulated $z$-depth magnitudes relative to the axis of rotation for both conditions, the distance between the 

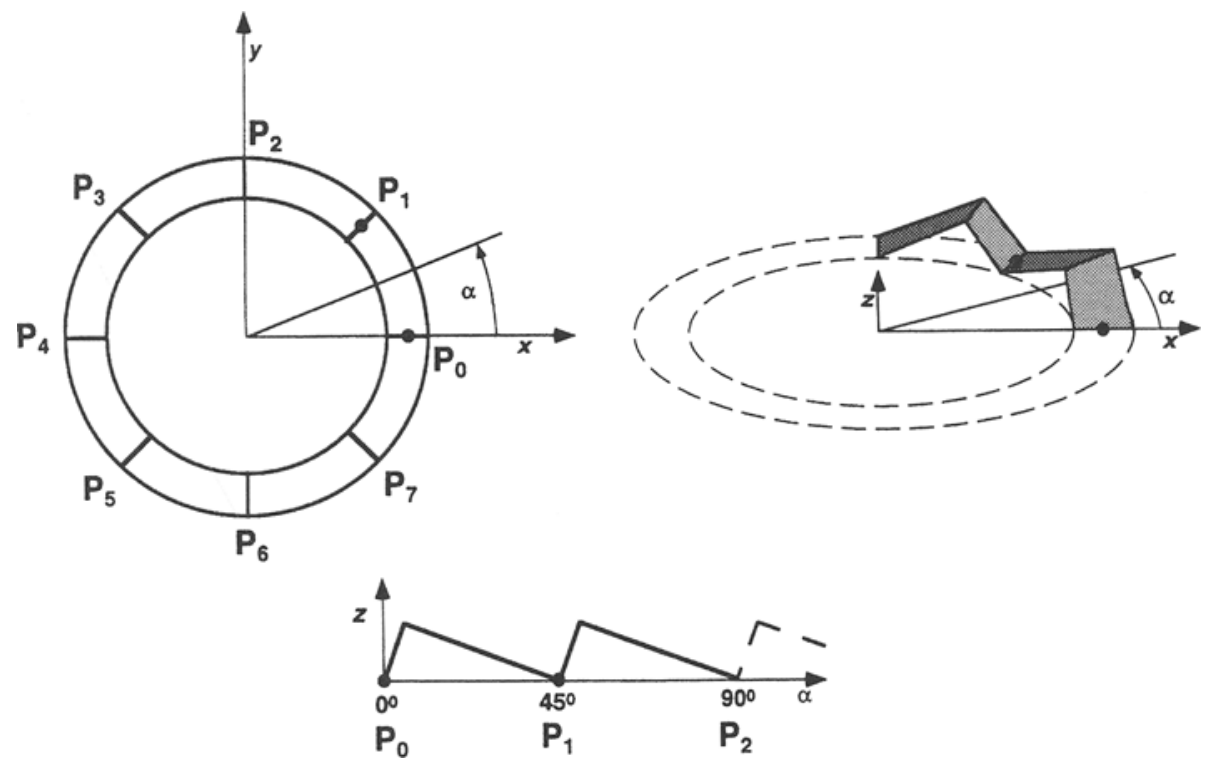

Figure 3. Schematic representation of the projection on the $x, y$ plane of a circular crown surface. Each of the eight portions of the surface contains a corrugation made up of two slanted surfaces. At the bottom are shown two corrugations in a graph in which the ordinate represents the $z$-map and the abscissa represents the angle $\alpha$. A schematic representation of two corrugations in the 3-D layout is provided at top right.

probe dots was set equal to $6.26 \mathrm{~cm}$ in the same-slant condition and to $5.24 \mathrm{~cm}$ in the different-slant condition.

The two surfaces underwent a rigid rotation through an angle of $\pm 5^{\circ}$. Rotation velocity of the two simulated surfaces varied sinusoidally with minima at the two extreme values of $x$. One oscillation cycle took 16 frames.

Procedure. All subjects were run individually in one session. They were instructed to judge the depth-order of the two probes. If they perceived the simulated dihedral angle as convex (concave), they were required to indicate which of the two probes (right or left) appeared closer (farther) in depth. In each trial, subjects indicated their choice with a keypress. Vision was monocular. Head motion was not restricted. While the experiment was run, the experimental room was dark. No restriction was placed on viewing time. No feedback was given until after the experiment was completed.

\section{Results and Discussion}

We transformed the frequency of reporting a correct response for each condition by an arcsine transformation (Winer, 1971). A 2 (same- vs. different-slant) $\times 2$ (left vs. right position) within-subjects analysis of variance (ANOVA) was run on the arcsine-transformed frequency data. The effect of the independent variable position was not significant $[F(1,9)=1.211$, n.s. $]$, nor was the interaction between condition and position $[F(1,9)=.015$, n.s.]. The effect of the variable condition, on the other hand, was significant $\left[F(1,9)=42.79, p<.001, \eta^{2}=.83\right]$ : In the same-slant condition, the depth-order relations were reported with $93.5 \%$ (left: $92 \%$, right: $95 \%$ ) accuracy, whereas in the different-slant condition, accuracy dropped to $18.5 \%$ (left: $15 \%$, right: $22 \%$ ).

Both these results are consistent with the predictions of the heuristic described by Equation 3 . As discussed in the introduction, in fact, the depth magnitudes recovered according to Equation 3 preserve the relative-depth relations for probe dots on surfaces with the same-slant magnitude. On the other hand, when the surfaces have different slants, Equation 3 predicts a relative underestimation of depth for the probe on the most slanted surface. In the present experiment, this underestimation overcame a depth difference of $8.6 \mathrm{~mm}$ in the opposite direction. In other words, the probe dot more distant from the axis of rotation appeared to be less distant from the axis of rotation. The heuristic of Equation 3, therefore, accounts for both the veridical performance in the same-slant condition and the below-chance performance in the different-slant condition. In the different-slant condition, the arcsinetransformed frequencies differed significantly from chance (the arcsine of .5) [ $t(9)=-2.95, p<.05]$.

It should also be noticed that the probe point $P_{1}$ (simulated on the most slanted surface) projected with a larger 2-D velocity than the probe point $\mathrm{P}_{0}$. Had the different projected velocities of the probe points given rise to a kinetic depth effect, we would have expected the largest projected 2-D velocity to be associated with the largest perceived distance from the axis of rotation. Instead, the results of this experiment indicate that, in most of the cases, the probe point $P_{1}$ was perceived as having the smallest distance from the axis of rotation. These data, therefore, indicate that the kinetic depth effect was overwhelmed by the local differences of the def component of the velocity field. In conclusion, the data confirm the hypothesis that motivated the present experiment: The ordinal structure perceived in SFM displays does not necessarily preserve the ordinal structure of the projected objects. 


\section{different-slant condition}

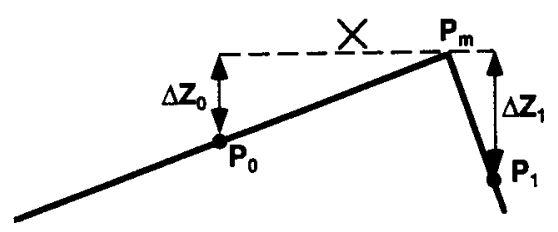

\section{same-slant condition}

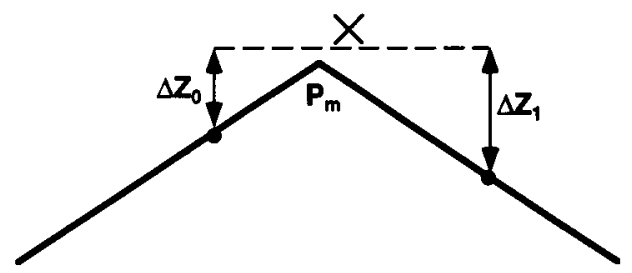

Figure 4. View from above of the simulated surface arrangements in the two experimental conditions of Experiment 1. The probe dots $P_{0}$ and $P_{1}$ have different simulated $z$-depths relative to the point $P_{m}\left(\Delta z_{0}<\Delta z_{1}\right)$.

\section{EXPERIMENT 2}

The aim of Experiment 2 was to extend the findings of Experiment 1 by employing smoothly curved surfaces rather than dihedral angles. In this experiment, the probe dots were placed at the same simulated distance from the axis of rotation (hence projecting the same 2-D velocity).

\section{Method}

Subjects. Twelve University of Trieste undergraduates participated in this experiment. All of them were naive to the purpose of the experiment.

Apparatus. The apparatus was the same as in Experiment 1.

Stimuli. The stimuli were the same as those of Experiment 1 except that the horizontal motions of the dots were computed as simulating an orthographic projection of a surface with a hyperbolic profile oscillating about a fixed vertical axis (Figure 5). The depth of each dot was determined by the equation of a hyperbola. At the midpoint of each oscillation cycle, the asymptotes of the simulated surface were slanted by 2.74 and $.36\left(70^{\circ}\right.$ and $\left.20^{\circ}\right)$. The side of the hyperbola with the greatest slant was either on the left (in half the trials) or on the right (in the other half) of the display. The two sides of the hyperbola were truncated at the same depth at their extremes. Two probe dots were depicted on the two sides of the hyperbolic surface at the same $z$-depth $(6 \mathrm{~cm})$ from the axis of rotation. The two probe dots, therefore, projected the same $2-D$ velocity. The position of the axis of rotation bisected evenly the length of the stimulus window. The hyperbolic surfaces underwent a rigid rotation through an angle of $\pm 15^{\circ}$. Rotational speed of the two simulated surfaces varied sinusoidally with minima at the two extreme values of $x$. One oscillation cycle took 20 frames. The stimulus window measured $13.55^{\circ}$ (width) $\times 1.29^{\circ}$ (height) of visual angle.

Design. Each subject viewed 20 presentations in random order of the two different stimuli (hyperbolic surfaces having the largest slant either on the right or on the left of the display). Eight additional trials were presented at the beginning of each experimental session in order to familiarize the subjects with the stimulus displays.

Procedure. Procedure and instructions were the same as in Experiment 1.

\section{Results and Discussion}

In $96.5 \%$ (left: $96 \%$, right: $97 \%$ ) of the cases, when the hyperbolic surfaces were perceived as concave (convex), subjects judged the probe dots simulated on the portion of the surfaces with the smaller slant to be closer (farther away). Subjects' responses of each were coded 1 if they were consistent with the predictions of Equation 3 and coded 0 otherwise. The arcsine-transformed frequencies of the responses of the subjects were significantly different from chance (the arcsine of .5) $[t(11)=13.124$, $p<.001]$. If ordinal structure had been preserved, we would not have expected a bias in either direction, since the two probe dots simulated the same relative-depth magnitudes. On the other hand, the bias exhibited by the subjects is predicted by the heuristic described by Equation 3: For probe dots simulating the same $z$-depth relative to the axis of rotation, larger magnitudes of relative depth are expected for the probe dot on the least slanted surface. It is important to notice, moreover, that in the present experiment different magnitudes of perceived
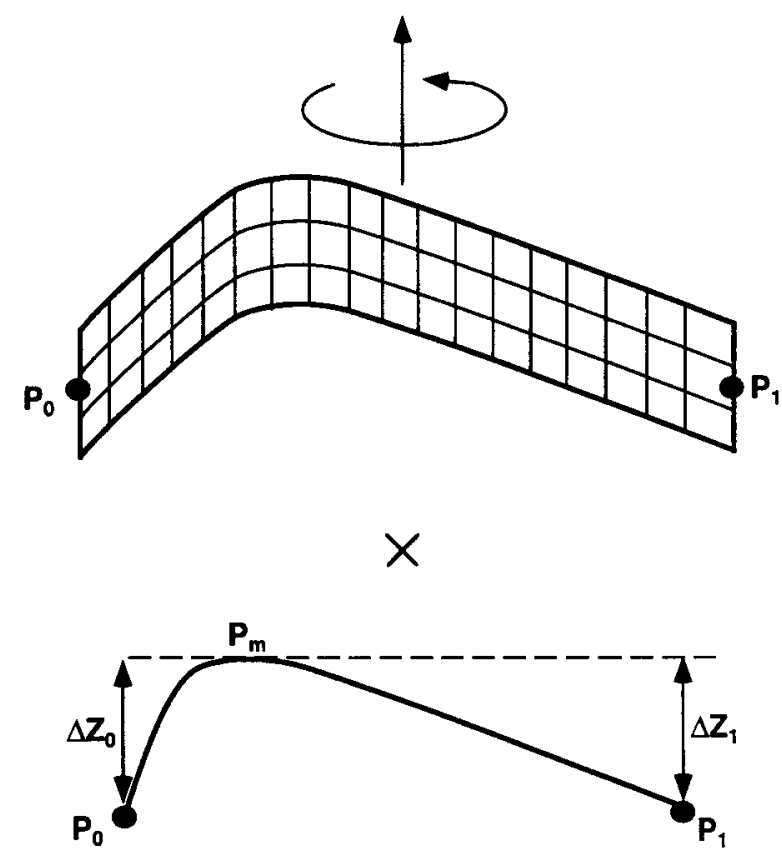

prediction: $\Delta \mathbf{Z}_{\mathbf{0}^{\prime}}<\Delta \mathbf{Z}^{\prime}{ }^{\prime}$

Figure 5. Schematic representation (top) and view from above (bottom) of the simulated surface used in Experiment 2. The probe dots $P_{0}$ and $P_{1}$ have the same simulated distance from the point $\mathbf{P}_{\mathrm{m}}\left(\Delta z_{0}=\Delta z_{1}\right)$. Equation 3 predicts that $\Delta z_{0}^{\prime}<\Delta z_{1}^{\prime}$. 


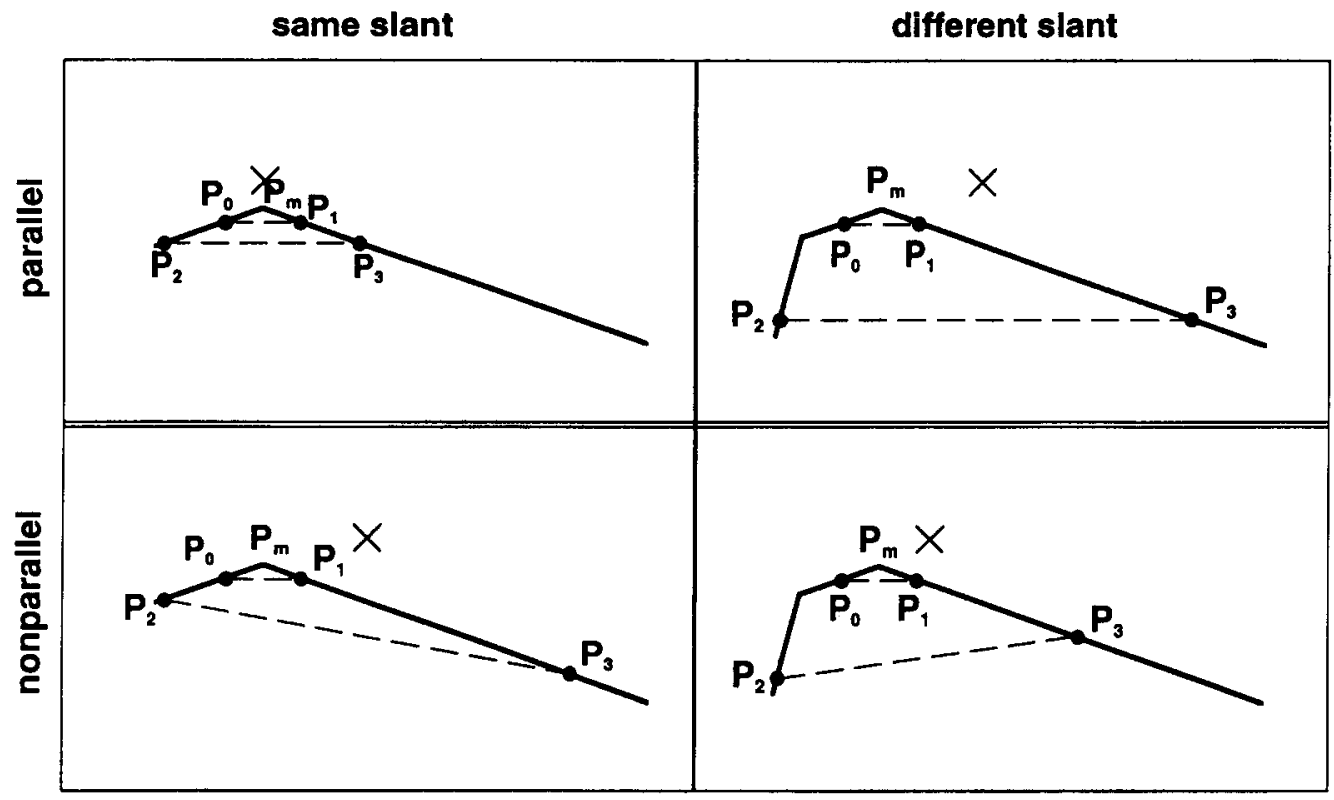

Figure 6. View from above of the simulated surface arrangements in the four conditions of Experiment 3. In the same-slant condition, the simulated surfaces have the same simulated slant. In the different-slant condition, the simulated surfaces have different simulated slants. In the parallel condition, the simulated $z$-depth magnitudes relative to the point $P_{m}$ are the same for $P_{0}$ and $P_{1}\left(\Delta z_{0}=\Delta z_{1}\right)$, and for $P_{2}$ and $P_{3}\left(\Delta z_{2}=\Delta z_{3}\right)$. In the nonparallel condition, the simulated $z$-depth magnitudes relative to the point $P_{m}$ are the same for $P_{0}$ and $P_{1}\left(\Delta z_{0}=\Delta z_{1}\right)$ and different for $P_{2}$ and $P_{3}\left(\Delta z_{2}<>\Delta z_{3}\right)$.

relative depth were assigned to the probe dots, even though they projected the same 2-D velocity. This indicates that even though the kinetic depth effect should have given rise to a constant amount of perceived relative depth, the distortions of perceived slant predicted by Equation 3 overwhelmed this effect, thus preventing the perceptual preservation of the depth-order relations of the projected object.

\section{EXPERIMENT 3}

An important property of both Euclidean and affine theories of depth recovery from motion is that parallelism is preserved: Lines that are parallel for the projected object also remain parallel in the recovered object. Moreover, parallel lines remain parallel for any affine transformation. The purpose of Experiment 3 was to investigate the perceptual preservation of parallelism. In particular, subjects were asked to discriminate between parallel and nonparallel (virtual) lines defined with respect to simulated surfaces having either same or different slants.

\section{Method}

Subjects. Nine University of Trieste undergraduates participated in this experiment. All of them were naive to the purpose of the experiment.

Apparatus. The apparatus was the same as in Experiment 1.

Design. Surface arrangement (displays made up of surfaces having either same or different slant magnitudes) and parallelism ( $z-$ depth locations for the probe dots identifying either truly parallel or nonparallel lines in 3-D) were the two independent variables. Each subject viewed 10 presentations of the four different stimuli in ran- dom order. Eight additional trials were presented at the beginning of each experimental session in order to familiarize the subjects with the stimulus displays.

Stimuli. The stimuli were the same as those of Experiment $1 \mathrm{ex}-$ cept that the horizontal motions of the dots were computed as simulating an orthographic projection of either two or three connected surfaces oscillating about a fixed axis (Figure 6). Four probe dots were depicted on two different surface arrangements specifying four conditions.

The same-slant stimuli simulated two surfaces with slants of .36 $\left(20^{\circ}\right)$ (Figure 6). Two pairs- $\left(\mathrm{P}_{0}, \mathrm{P}_{1}\right)$ and $\left(\mathrm{P}_{2}, \mathrm{P}_{3}\right)$-of probe dots were depicted on the simulated surfaces with different $y$-coordinates. The probe pair $\left(\mathrm{P}_{0}, \mathrm{P}_{1}\right)$ was depicted on the two surfaces at a distance of $1 \mathrm{~cm}$ from the rotation axis for both dots. The probe pair $\left(\mathrm{P}_{2}, \mathrm{P}_{3}\right)$ was depicted on the simulated surfaces either at the same distance relative to the rotation axis ( $4 \mathrm{~cm}$; parallel condition) or at different distances ( 2.5 and $4 \mathrm{~cm}$; nonparallel condition).

The different-slant stimuli simulated two surfaces with a slant of $.36\left(20^{\circ}\right)$ and one surface with a slant of $3.72\left(75^{\circ}\right.$; see Figure 6). The probe pair $\left(\mathrm{P}_{0}, \mathrm{P}_{1}\right)$ was depicted on the .36 surfaces, whereas the probe pair $\left(\mathrm{P}_{2}, \mathrm{P}_{3}\right)$ was depicted on the surfaces with slants of .36 and 3.72. The dots of the probe pair $\left(\mathrm{P}_{0}, \mathrm{P}_{1}\right)$ were simulated with the same depth relative to the rotation axis $(1 \mathrm{~cm})$. The dots of the probe pair $\left(\mathrm{P}_{2}, \mathrm{P}_{3}\right)$ were positioned either at the same depth relative to the rotation axis ( $4 \mathrm{~cm}$; parallel condition) or at different depths ( 2.5 and $4 \mathrm{~cm}$; nonparallel condition). The difference of $1.5 \mathrm{~cm}$ in the simulated $z$-depth magnitudes for the dots of pair $\left(\mathrm{P}_{2}, \mathrm{P}_{3}\right)$ was determined through a pilot experiment so that the (virtual) lines in the different-slant nonparallel condition would appear as parallel.

The stimuli were presented in random order. The position of the axis of rotation bisected the $x$-extent of the probe dots. The connected surfaces underwent a rigid rotation through an angle of $\pm 3^{\circ}$. Rotation velocity of the simulated surfaces varied sinusoidally with minima at the two extreme values of $x$; one oscillation cycle took 16 frames. 
Procedure. The subjects were told to consider two virtual straight lines, one of them connecting the dots of probe pair $\left(\mathrm{P}_{0}, \mathrm{P}_{1}\right)$ and the other connecting the dots of probe pair $\left(\mathrm{P}_{2}, \mathrm{P}_{3}\right)$. They were told that these virtual lines always projected two parallel lines on the projection plane, even though they could be parallel or not in 3-D space depending on the $z$-coordinates of the probe dots. The subjects were told that the two virtual lines were simulated as parallel in 3-D space in half the trials. The subjects' task was to decide, on each trial, if the two lines appeared to be parallel or not in 3-D space. Otherwise, the procedure was the same as in Experiment 1.

\section{Results and Discussion}

The percentages of correct responses were $83 \%$ (sameslant parallel condition), $78 \%$ (same-slant nonparallel condition), $34 \%$ (different-slant parallel condition), and $42 \%$ (different-slant nonparallel condition). A 2 (samevs. different-slant) $\times 2$ (parallel vs. nonparallel) withinsubjects ANOVA was run on the arcsine-transformed frequency of reporting a correct response in each condition. The effect of surface arrangement was significant $[F(1,8)=$ $\left.34.21, p<.001, \eta^{2}=.81\right]$; in the same-slant condition, percent correct was $80.5 \%$, whereas in the different-slant condition, it was $38 \%$. The effect of parallelism was not significant $[F(1,8)=.147$, n.s. $]$, and neither was the interaction between the two independent variables $[F(1,8)=$ .361, n.s.].

As for the previous experiments, the present findings are consistent with the predictions of Equation 3. In the same-slant condition, in fact, the relative-depth magnitudes of the probe pairs $\left(\mathrm{P}_{0}, \mathrm{P}_{1}\right)$ and $\left(\mathrm{P}_{2}, \mathrm{P}_{3}\right)$ recovered according to Equation 3 were affected by the same amount of error. As a consequence, Equation 3 predicts a veridical performance in this condition. Percent correct was, in fact, $80.5 \%$. In the different-slant condition, on the other hand, the relative-depth magnitudes of the probe pairs $\left(\mathrm{P}_{0}, \mathrm{P}_{1}\right)$ and $\left(\mathrm{P}_{2}, \mathrm{P}_{3}\right)$ recovered according to Equation 3 were affected by different amounts of error. As a consequence, Equation 3 predicts a nonveridical performance in this condition. In fact, percent correct was $35 \%$. In the different-slant parallel condition, subjects tended to judge truly parallel virtual lines as nonparallel $[t(8)=$ $-3.07, p<.05$ ]; in the different-slant nonparallel condition, by contrast, performance was at chance $[t(8)=$ $-.87, \mathrm{n} . \mathrm{s}$.$] . In conclusion, the data confirm the hypoth-$ esis that motivated the present experiment: The parallelism of lines defined by features of the projected objects is not necessarily preserved in the 3-D shape perceived in SFM displays.

\section{EXPERIMENT 4}

For both Euclidean and affine theories of depth recovery from motion, a necessary condition for a derived surface $z(x, y)$ is the vanishing of the algebraic sum of the depth differences between adjacent couples of points on a close curve in the projection. If this condition is not satisfied, the points cannot be interpreted as belonging to a close curve in the projection of a surface. This con- straint was tested by measuring the perceived depth-order relations of dot probes positioned on oscillating corrugated circular crown surfaces. Since the slant recovered by the heuristic described by Equation 3 is not linearly related to projected slant, we hypothesized that the subjects' depth-order judgments would reveal a bias incompatible with an internally consistent 3-D structure.

\section{Method}

Subjects. Twelve University of Trieste undergraduates participated in this experiment. All of them were naive to the purpose of the experiment.

Design. Each subject viewed five presentations in random order of eight different stimuli (corrugated circular crown surfaces with a pair of probe dots positioned in correspondence with each of the eight corrugations of the surface). Eight additional trials were presented at the beginning of each experimental session in order to familiarize the subjects with the stimulus displays.

Stimuli. The stimuli were high-luminance dots moving on a lowluminance background. The horizontal motions of the dots were computed as simulating an orthographic projection of a corrugated circular crown surface oscillating about a fixed vertical axis. There were eight corrugations. Each corrugation was made up of two surfaces slanted 2.74 and $.36\left(20^{\circ}\right.$ and $\left.70^{\circ}\right)$ (see Figure 3). The external and internal diameters of the circular crown measured $12.49^{\circ}$ and $9.65^{\circ}$ of visual angle. Two probe dots were positioned at two contiguous peaks of the corrugation. Eight pairs of probe dots were used-one for each pair of contiguous planar surfaces making up the circular crown surface. Only one pair of probe dots was presented in each trial.

The dots of the random-dot surface were randomly distributed with uniform probability density over the projection plane (not evenly distributed over the simulated surface). Average dot density was equal to $4 \mathrm{dots} / \mathrm{cm}^{2}$. The circular crown surface underwent a rigid rotation through an angle of $\pm 8^{\circ}$. Rotational speed varied sinusoidally with minima at the two extreme values of $x$. One oscillation cycle took 40 frames.

Procedure. Procedure and instructions were the same as in Experiment 1.

\section{Results and Discussion}

A one-way within-subjects ANOVA run on the arcsinetransformed frequencies of reporting a response in the direction predicted by Equation 3 did not reveal a significant effect of the tilt of the corrugations $[F(7,5)=1.0$, n.s.]. Regardless of tilt, however, in the $86.04 \%$ of the cases subjects judged as closer (farther away) the probe simulated on the least slanted of the two sides of the corrugations of the circular crown surface when the corrugations were viewed as concave (convex). This bias was well above chance $[t(11)=10.188, p<.001]$.

As for Experiment 2, the bias exhibited by the subjects is consistent with the predictions of Equation 3: For probe dots simulating the same $z$-depth magnitude relative to the axis of rotation, subjects tended to perceive a larger relative depth for the probe dots on the least slanted surface of the corrugations. Since the probe dots were placed on a close path on the simulated surface, this bias suggests that the local signing of the perceived depth-order relations is incompatible with an internally consistent $3-\mathrm{D}$ structure. 


\section{GENERAL DISCUSSION}

Four general conclusions can be drawn from the results of the present experiments. (1) The perceptual signing of the depth-order relations does not necessarily preserve the ordinal structure of the projected objects. In Experiment 1 , in fact, we found that subjects judged veridically the depth-order relations when two probe dots were placed on rigidly oscillating surfaces having the same slant magnitudes, but provided systematically distorted judgments when two probe dots were placed on planar surfaces having different slant magnitudes. Moreover, in Experiment 2 we found that when asked to judge the relative depth order of a pair of probe dots, subjects were biased toward judging as closer the probe dot embedded in the velocity field with the smaller def, even if the two probe dots simulated the same depth magnitude (i.e., projected identical 2-D velocities). (2) Projected lines that are truly parallel in 3-D space are not necessarily perceived to be parallel (i.e., perceived 3-D shape does not preserve 3-D parallelism). In Experiment 3, in fact, we found that subjects veridically discriminated parallel from nonparallel (virtual) lines connecting four probe dots in 3-D space when the probe dots were simulated as lying on planar surfaces with the same slant. Conversely, subjects were not able to perform the experimental task at better than chance level when the probe dots were placed on rigidly oscillating surfaces with different slants. (3) The integration of the local judgments of depth-order relations along a closed path does not necessarily vanish (i.e., perceived 3-D shape is not internally consistent). In Experiment 4, in fact, we found that when asked to judge the relative depth order of pairs of probe dots, subjects were biased toward judging as closer the probe dot embedded in the velocity field with the smaller $d e f$, even if the two probe dots simulated the same depth magnitude. Since the pairs of probe dots were positioned on a closed path on the simulated 3-D surface, this bias made the local judgments of the depth order relations inconsistent with the properties of a 3-D shape derived by any Euclidean or affine model of depth recovery from motion. (4) The perceived relative-depth magnitudes of probe dots in SFM displays do not depend solely on their projected 2-D velocities. In Experiments 2 and 4, in fact, we found that subjects reliably associated different relative-depth magnitudes with probe dots projecting the same 2-D velocity. Moreover, in Experiment 1 we found that smaller relativedepth magnitudes were associated with probe dots projecting larger 2-D velocities. These findings suggest, therefore, that the perceived relative-depth magnitude of a target point in an SFM display should not be understood as a linear function of its projected 2-D velocity, but rather as a function of the def component of the velocity field constituting the projected 2-D velocity of the target point.

Especially troublesome for any Euclidean or affine model of depth recovery from motion are the results of Experiment 4. These results, in fact, suggest that perceived depth-order relations are internally inconsistent, thus making the properties of the perceived 3-D shape incompatible with the properties of both Euclidean or affine geometries. Other evidence besides the present results suggests that perceived 3-D shape is not internally consistent. In the shape-from-shading literature, a similar result has been reported by Todd and Reichel (1989). In the SFM literature, Domini and Braunstein (1998) obtained a similar result in a study of perceived depth. Domini and Braunstein placed two pairs of probes- $\left(\mathrm{P}_{0}, \mathrm{P}_{1}\right)$ and $\left(\mathrm{P}_{2}, \mathrm{P}_{3}\right)$-on different regions of a curved surface and predicted that judged depth separation would vary with the mean slant of the regions on which the probes were placed for constant simulated depth separations and constant simulated angular velocities. In their stimuli, the pairs of dots $\left(\mathrm{P}_{0}, \mathrm{P}_{1}\right)$ and $\left(\mathrm{P}_{2}, \mathrm{P}_{3}\right)$ had the same depth separation, as $\operatorname{did}\left(\mathrm{P}_{1}, \mathrm{P}_{2}\right)$ and $\left(\mathrm{P}_{3}, \mathrm{P}_{0}\right)$. However, the pairs $\left(\mathrm{P}_{0}, \mathrm{P}_{1}\right)$ and $\left(\mathrm{P}_{2}, \mathrm{P}_{3}\right)$ lay on patches having different slants, whereas the pairs $\left(\mathrm{P}_{0}, \mathrm{P}_{3}\right)$ and $\left(\mathrm{P}_{1}, \mathrm{P}_{2}\right)$ lay on patches having the same slants. They found that subjects veridically judged the perceived depth separations of the pairs $\left(\mathrm{P}_{0}, \mathrm{P}_{3}\right)$ and $\left(\mathrm{P}_{1}, \mathrm{P}_{2}\right)$ to be the same since both pairs lay on regions of the curved surface with identical slants. However, subjects mistakenly judged the perceived depth separations of the pairs $\left(\mathrm{P}_{0}, \mathrm{P}_{1}\right)$ and $\left(\mathrm{P}_{3}, \mathrm{P}_{2}\right)$ to be different since both pairs lay on regions of the curved surface with different slants. Therefore, the sum $z_{01}^{\prime}+$ $z_{12}^{\prime}$ was not equal to $z_{23}^{\prime}+z_{30}^{\prime}$, since $z_{01}^{\prime}>z_{23}^{\prime}$ and $z_{12}^{\prime}=z_{30}^{\prime}$. Consistent with the findings of our Experiment 4, hence, Domini and Braunstein found that the metric judgments were internally inconsistent since the algebraic sum of the depth judgments along a closed path was not zero.

The present results are consistent with the view that human perception of SFM is based on an analysis of the first-order optic flow (e.g., Todd \& Bressan, 1990; Todd \& Norman, 1991; Todd et al., 1988). Our results, however, suggest that the perceptual analysis of the first-order optic flow is not necessarily veridical. Whereas the psychophysical tasks employed in the present research could be performed veridically by using solely the first-order relations of the optic flow, we found that perceptual performance was systematically distorted. These systematic distortions have been interpreted as the consequence of a heuristic (rather than a geometrically correct) analysis of the first-order optic flow.

The hypothesis that the perceptual system makes use of specific heuristic processes to derive 3-D motion and shape from moving 2-D images has recently been proposed by several investigators (Braunstein, 1994; Caudek \& Proffitt, 1993; Domini \& Caudek, in press; Domini, Caudek, \& Proffitt, 1997; Liter \& Braunstein, 1998; Liter et al., 1993; Pollick, Nishida, Koike, \& Kawato, 1994). In our previous research, we showed that many aspects of perceived SFM may be accounted for by a heuristic analysis based on def. Domini et al. (1997) found that the variance of the deformation of the individual triplets of image features ${ }^{3}$ influences the discrimination between rigid and nonrigid motion. Displays with a low variance of the deformation tended to be judged as rigid, and those with a 
high variance of the deformation tended to be judged as nonrigid, regardless of whether they simulated a mathematically correct projection of a rigid motion or not. In another set of experiments, Domini et al. (1997) found that regardless of the magnitudes of simulated angular rotation (in multiview displays), perceived rotation was a monotonically increasing function of def. Domini, Caudek, Turner, and Favretto (1998) found that the judgments of "constant" or "variable" angular velocity were only slightly influenced by the projected angular velocities, but were greatly affected by the variations of $d e f$. Caudek and Domini (in press) found that the perceived slant of the axis of rotation was influenced by the ratio between def and the component of the global velocity vector parallel to the image plane. When this ratio was held constant in each frame transition of the stimulus sequence, the stimuli tended to be judged as fixed-axis rotations. Conversely, when the ratio varied, the stimuli tended to be judged as nonfixed-axis rotations. These relations held regardless of whether the stimuli simulated fixed-axis rotations or not. The present experiments were intended to contribute to this body of research by showing how a heuristic analysis deriving perceived surface slant from the information available in two views is capable (for the tasks and within the stimulus parameters used here) of accounting for both veridical performance and systematically distorted depth-order judgments.

It is important to point out that the violations of Euclidean and affine properties previously highlighted have been found in very restrictive stimulus conditions: SFM displays in which texture density was controlled and all other cues to depth were removed. Moreover, our stimuli simulated small angular rotations, thus preventing the second-order properties of the optic flow from being perceptually salient. The present findings, therefore, cannot be generalized beyond the specific stimulus conditions studied here. It remains an issue for future research to establish whether or not the perceptual derivation of 3-D shape preserves the Euclidean and affine properties for full-cue conditions and large displacements between the observer and distal objects. An alternative interpretation of the present findings, in fact, could attribute the sublinear mapping between $d e f$ and surface slant to the presence of a variety of interfering sources of information. In our displays, several cues beyond the velocity gradients could have contributed to perceived 3-D shape (e.g., cues to flatness provided by the homogeneous texture density, accommodation cues, and such top-down influences as, perhaps, a cognitive default toward vertical symmetry of 3-D shape). As a consequence, one might argue that the apparent sublinearity found in the previous experiments could reside in systems beyond the SFM system, as, for example, a more general slant perception mechanism that combines all sources of information specifying 3-D shape ${ }^{4}$ Germane to this issue is a recent contribution provided by Norman, Todd, Perotti, and Tittle (1996), who found, however, that the Euclidean properties are also perceptually distorted in the case of full-cue environments providing converging sources of information about 3-D shape.

\section{REFERENCES}

Bennett, B. M., Hoffman, D. D., Nicola, J. E., \& Prakash, C. (1989). Structure from two orthographic views of rigid motion. Journal of the Optical Society of America A, 6, 1052-1069.

BRAUnSTEIN, M. L. (1989). Structure from motion. In J. I. Elkind, S. K. Card, J. Hochberg, \& B. M. Huey (Eds.), Human performance for computer aided engineering (pp. 189-205). Washington, DC: National Academy Press.

Braunstein, M. L. (1994). Decoding principles, heuristics and inference in visual perception. In G. Jansson, S. S. Bergstrom, \& W. Epstein (Eds.), Perceiving events and objects (pp. 436-446). Hillsdale, NJ: Erlbaum.

Braunstein, M. L., Hoffman, D. D., Shapiro, L. R., Andersen, G. J., \& BENNETT, B. M. (1987). Minimum points and views for the recovery of three-dimensional structure. Journal of Experimental Psychology: Human Perception \& Performance, 13, 335-343.

CAUdek, C., \& Domini, F. (in press). Perceived orientation of axis of rotation in structure-from-motion. Journal of Experimental Psychology: Human Perception \& Performance.

CAudek, C., \& Proffit , D. R. (1993). Depth perception in motion parallax and stereokinesis. Journal of Experimental Psychology: Human Perception \& Performance, 19, 32-47.

Cutring, J. E., Bruno, N., Brady, N. P., \& Moore, C. (1992). Selectivity, scope, and simplicity of models: A lesson from fitting judgments of perceived depth. Journal of Experimental Psychology: General, 121, 364-381.

Domini, F., \& Braunstein, M. L. (1998). 3D structure from motion is neither Euclidean nor affine. Journal of Experimental Psychology: Human Perception \& Performance, 24, 1273-1295.

Domini, F., \& Caudek, C. (in press). Perceiving surface slant from deformation of optic flow. Journal of Experimental Psychology: Human Perception \& Performance.

Domini, F., Caudek, C., \& Gerbino, W. (1995). Perception of surface attitude in SFM displays. Investigative Ophthalmology \& Visual Science, 36, $\mathrm{S} 360$.

Domini, F., Caudek, C., \& Proffitt, D. R. (1997). Misperceptions of angular velocities influence the perception of rigidity in the Kinetic Depth Effect. Journal of Experimental Psychology: Human Perception \& Performance, 23, 1111-1129.

Domini, F., Caudek, C., Turner, J., \& Favretto, A. (1998). Discriminating constant from variable angular velocities in the kinetic depth effect. Perception \& Psychophysics, 60, 747-760.

EAGLE, R. A., \& BlaKe, A. (1995). Two-dimensional constraints on three-dimensional structure from motion tasks. Vision Research, 35. 2927-2941.

Hildreth, E. C., Grzywacz, N. M., Adelson, E. H., \& Inida, V. K. (1990). The perceptual buildup of three-dimensional structure from motion. Perception \& Psychophysics, 48, 19-36.

HofFMAN, D. D. (1982). Inferring local surface orientation from motion fields. Journal of the Optical Society of America A, 72, 888-892. Koenderink, J. J. (1986). Optic flow. Vision Research, 26, 161-180. KoENDERINK, J. J., \& VAN DOORN, A. J. (1975). Invariant properties of the motion parallax field due to the movement of rigid bodies relative to an observer. Optica Acta, 22, 773-791.

Koenderink, J. J., \& VAN DoORN, A. J. (1976). Local structure of movement parallax of the plane. Journal of the Optical Society of America A, 66, 717-723.

Koenderink, J. J., \& van DoORn, A. J. (1991). Affine structure from motion. Journal of the Optical Society of America A, 8, 377-385.

LiTER, J. C., \& BRAUNSTEIN, M. L. (1998). The relationship of vertical and horizontal velocity gradients in the perception of shape, rotation, 
and rigidity. Journal of Experimental Psychology: Human Perception \& Performance, 24, 1257-1272.

Liter, J. C., Braunstein, M. L., \& Hoffman, D. D. (1993). Inferring structure from motion in two-view and multiview displays. Perception, 22, 1441-1465.

Longuet-Higgins, H. C., \& Prazdny, K. (1980). The interpretation of a moving retinal image. Proceedings of the Royal Society of London: Series $B, 208,385-397$.

MILES, W. R. (1931). Movement interpretations of the silhouette of a revolving fan. American Journal of Psychology, 43, 392-405.

MusatTI, C. L. (1924). Sui fenomeni stereocinetici [On stereokinetic phenomena]. Archivio Italiano di Psicologia, 6, 205-219.

Norman, J. F., \& ToDD, J. T. (1992). The visual perception of 3-D dimensional form. In G. A. Carpenter \& S. Grossberg (Eds.), Neural networks for vision and image processing (pp. 93-110). Cambridge, MA: MIT Press.

Norman, J. F., \& TodD, J. T. (1993). The perceptual analysis of structure from motion for rotating objects undergoing affine stretching transformations. Perception \& Psychophysics, 53, 279-291.

Norman, J. F., Todd, J. T., Perotti, V. J., \& Tittle, J. S. (1996). The visual perception of three-dimensional length. Journal of Experimental Psychology: Human Perception \& Performance, 22, 173-186.

Pollick, F. E. (1997). The perception of motion and structure in structure-from-motion: Comparison of affine and Euclidean formulations. Vision Research, 37, 447-466.

Pollick, F. E., Nishida, S., Koike, Y., \& Kawato, M. (1994). Perceived motion in structure from motion: Pointing responses to the axis of rotation. Perception \& Psychophysics, 56, 91-109.

Prazdny, K. (1980). Egomotion and relative depth map from optical flow. Biological Cybernetics, 36, 87-102.

Todd, J. T., AKerstrom, R. A., Reichel, F. D., \& Hayes, W. (1988). Apparent rotation in three-dimensional space: Effects of temporal, spatial, and structural factors. Perception \& Psychophysics, 43, 179-188.

TODD, J. T., \& BRESSAN, P. (1990). The perception of 3-dimensional affine structure from minimal apparent motion sequences. Perception \& Psychophysics, 48, 419-304.

ToDD, J. T., \& NoRMAN, J. F. (1991). The visual perception of smoothly curved surfaces from minimal apparent motion sequences. Perception \& Psychophysics, 50, 509-523.

TODD, J. T., \& REICHEL, F. D. (1989). Ordinal structure in the visual perception and cognition of smoothly curved surfaces. Psychological Review, 96, 643-657.

Ullman, S. (1979). The interpretation of visual motion. Cambridge, MA: MIT Press.

Ullman, S. (1983). The interpretation of structure from motion. Proceedings of the Royal Society of London: Series B, 203, 405-426.

ULLMAN, S. (1984). Maximizing rigidity: The incremental recovery of 3-D structure from rigid and non-rigid motion. Perception, 13, 255-274.

Wallach, H., \& O'Connell, D. N. (1953). The kinetic depth effect. Journal of Experimental Psychology, 45, 205-217.

WINER, B. J. (1971). Statistical principles in experimental design (2nd ed.). New York: McGraw-Hill.

\section{NOTES}

1. The scope of the present investigation is restricted to the case of an orthographic projection since any perspective projection can be locally approximated by an orthographic projection.

2. In the present experiments, we did not directly test the model $\sigma^{\prime}=f_{\text {sublinear }}(d e f)$ since we didn't manipulate $\omega$. The scope of the present investigations was restricted to the study of the implications of this model.

3. The velocity vectors associated with three points provide the minimum conditions for computing def.

4. For the issue of information integration in depth perception, see, for example, Cutting, Bruno, Brady, and Moore (1992).

\section{APPENDIX}

A planar surface can be described as

$$
f(x, y)=g_{1} x+g_{2} y+d,
$$

where $g_{1}$ and $g_{2}$ are the two components of the depth gradient of the plane in the $x$ and $y$ directions. The orientation of a planar patch in 3-D space can be described in terms of its slant $(\sigma)$ and tilt $(\tau)$. Slant is defined as the tangent of the angle between the line of sight (i.e., the $z$-axis) and the normal to the patch. Tilt is defined as the tangent of the angle between the projection of the normal to the patch and the $x$-axis. Slant and tilt can be calculated as a function of the two components of the depth gradient $g_{1}$ and $g_{2}$ :

$$
\sigma=\sqrt{g_{1}^{2}+g_{2}^{2}}, \quad \tau=\frac{g_{2}}{g_{1}} .
$$

Previous empirical evidence suggests that the perceived slant $\left(\sigma^{\prime}\right)$ of a rotating planar surface is a monotonically increasing function of $d e f$ and that tilt $\left(\tau^{\prime}\right)$ is correctly derived (Domini et al., 1995, and Domini and Caudek, in press):

$$
\begin{gathered}
\sigma^{\prime}=f(\text { def }) ; \\
\tau^{\prime}=\tau .
\end{gathered}
$$

In this Appendix, we will consider the implications that these hypotheses have for the derivation of depth separation of two dots located on a planar surface. The first point $P_{0}$ will be located at the origin of a Cartesian system, and a second point $P_{1}$ will have a generic position $(x, y, z)$. There are infinite planar surfaces passing through the points $P_{0}$ and $P_{1}$. Each of these surfaces is defined by the equation:

$$
z=g_{1} x+g_{2} y .
$$

By combining Equations A2 we obtain

$$
\sigma=\left|g_{1}\right| \sqrt{1+\tau^{2}} .
$$

Since tilt is defined as the ratio between $g_{2}$ and $g_{1}$, and the slant is defined as the sum of the square of the two components of the def gradient, the separation in depth between the points $\mathrm{P}_{0}$ and $P_{1}$ becomes

$$
z=\frac{\sigma}{\sqrt{1+\tau^{2}}}(x+\tau y)
$$

By taking into account Equations A3 and A4, the derived separation in depth can be expressed as function of def, the simulated depth separation, and the angular velocity $\omega$ :

$$
z^{\prime}=z \omega \frac{f(d e f)}{d e f} .
$$

In conclusion, if the function $f(d e f)$ is a sublinear function of def, then the ratio between $f(d e f)$ and def is a decreasing function $(F(d e f))$ of def. Equation A8 can be therefore rewritten as

$$
z^{\prime}=z \omega F(\operatorname{def})
$$

(Manuscript received February 26, 1997; revision accepted for publication September 18, 1997.) 